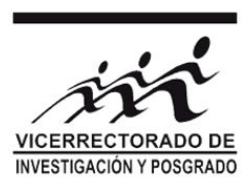

\title{
Formulación hamiltoniana de la métrica de Friedmann
}

\author{
Fulgencio Villegas ${ }^{* 1}$ y Teófilo Vargas ${ }^{* * 1}$ \\ ${ }^{1}$ Universidad Nacional Mayor de San Marcos, Ap. Postal 14-0149, Lima, Perú
}

Recibido 03 agosto 2018 - Aceptado 15 octubre 2018

\begin{abstract}
Partiendo del modelo cosmológico de Friedmann, en el cual se ha considerado la función de lapse $N(t)$ y la función de escala $a(t)$, se determina el escalar de curvatura de Ricci, el tensor de curvatura extrínseca, y a partir de ello se determina el lagrangiano total formado por el lagrangiano gravitacional y el de materia; y a partir de los momentos conjugados, finalmente, se construye el hamiltoniano de dicho modelo cosmológico. Palabras clave: Relatividad general, gravitación, cosmología, métrica FRW.
\end{abstract}

\section{Hamiltonian formulation of the metric of Friedmann}

From the cosmological Friedmann model, which has been considered function lapse $N(t)$ and the scaling function $a(t)$, it is determined the scalar curvature of Ricci tensor extrinsic curvature, and from this the total formed by the gravitational Lagrangian Lagrangian and the matter is determined; and from conjugates finally time, the Hamiltonian of the cosmological model is built.

Keywords: General Relativity, gravitation, cosmology, FRW metric.

\section{Introducción}

La cosmología es el estudio del universo considerado como un todo. El modelo estándar de la cosmología empezó a ser constituido por Einstein en 1917 poco después de haber publicado su trabajo sobre relatividad general [1]. El primer modelo cosmológico relativista fue propuesto por Einstein [2], este modelo poseía características de ser homogéneo, isotrópico, finito y estático. En el mismo año, el astrónomo holandés Willem de Sitter publicó un trabajo [3] que consistía en una nueva solución de la relatividad general incluyendo la constante cosmológica. El estudio de un universo en expansión se debe a Alexander Friedmann [4] quien en su artículo publicado en 1922 considera un espacio con curvatura constante y positiva obteniendo, por primera vez, soluciones a las ecuaciones de Einstein con características expansionistas con o sin constante cosmológica.

La formulación hamiltoniana de un modelo cosmológico tiene, en la actualidad, particular importancia por ser una via para concretar la cuantización de la gravedad y la cosmología. La formulación hamiltoniana de la relatividad general se ha desarrollado principalmente en los artículos de Dirac [5] y Arnowit, Deser and Miener (ADM) [6]. Esta formulación fue aplicada a los modelos cosmológicos clásicos incluidos los de Bianchi, dando lugar a la llamada
Cosmología Hamiltoniana [7].

La importancia del enfoque hamiltoniano para la métrica de Friedmann radica en que los resultados nos permite entender la dinámica y energía del universo, asimismo, permitirá analizar la densidad de energía en el universo y su relación con la velocidad de expansión, su relación con el factor de escala $a(t)$ y también permitirá analizar la termodinámica de la expansión del universo.

Este trabajo está basado en la formulación ADM y nuestro objetivo es calcular la formulación Hamiltoniana de la métrica de Friedmann, previamente calculamos la curvatura extrínseca y la formulación Lagrangiana total.

\section{Formulación ADM de la Relatividad General}

Una manera de analizar la dinámica de la relatividad general consiste en considerarla como un problema de Cauchy, es decir, analizar la dinámica como la evolución de una hipersuperficie tridimensional donde estén definidos los campos (formalismo ADM). Esta manera de formular la relatividad general fue desarrollada por $\mathrm{R}$. Arnowitt, S. Deser y C. W. Misner [8].

Para construir la formulación de la relatividad general, debemos foliar el espacio-tiempo, definido por una variedad diferenciable $\mathrm{M}$ y una métrica $g_{\mu \nu}$ en hipersuperficies

\footnotetext{
*fvillegass@unmsm.edu.pe

** teofilo.vargas@gmail.com
} 
espaciales $\sum$ en el cual esta definida una métrica espacial $h_{i j}$ y una curvatura extrínseca $k_{\mu \nu}$. El par $\left(\mathrm{M}, g_{\mu \nu}\right)$ satisface las ecuaciones de Einstein [9]. Debido a que la foliación puede ser arbitraria, entonces el parámetro t no necesariamente es el tiempo propio, es un parámetro sin significado físico, simplemente es una etiqueta para cada folio o rebanada. Introduciremos una variable denominada función lapse $\mathrm{N}$ que mide la variación del tiempo propio con el parámetro $t$, es decir:

$$
\frac{d \tau}{d t}=N
$$

Por lo tanto un elemento de linea podemos escribirlo como:

$$
d s^{2}=-N^{2} d t^{2}+h_{i j} d x^{i} d x^{j}
$$

La curvatura extrínseca viene expresada por:

$$
k_{i j}=\frac{1}{2 N} h_{i j}
$$

y el escalar de curvatura en términos de la curvatura extrínseca viene dado por:

$$
R=R^{3}-K^{2}+k_{i j} k_{i j}
$$

donde $R^{3}$ es el escalar de curvatura definido en la hipersuperficie.

\section{La métrica de Friedmann}

En la teoría gravitacional de Einstein [9], la gravedad viene descrita por la métrica del espacio-tiempo $g_{\mu \nu}$. El elemento de línea infinitesimal e invariante $d s^{2}$ esta dado por:

$$
d s^{2}=g_{\mu \nu} d x^{\mu} d x^{\nu}
$$

Einstein utilizó la geometría diferencial para determinar el tensor de curvatura y el escalar de curvatura y así determinar del tensor métrico. En forma modificada, las ecuaciones de Einstein incluyendo la constante cosmológica $\Lambda$ presentan la forma:

$$
R_{\mu \nu}-\frac{1}{2} R g_{\mu \nu}+\Lambda g_{\mu \nu}=8 \pi G T_{\mu \nu},
$$

donde $G$ es la constante gravitacional de Newton y $T_{\mu \nu}$ es el tensor de energía-momentum. Las ecuaciones de Einstein representan 10 ecuaciones diferenciales acopladas. Con la suposición de Friedmann-Lemaitre-RobertsonWalker (FLRW) sobre el principio cosmológico la métrica se representa como [10]:

$$
d s^{2}=-d t^{2}+a^{2}(t) \frac{d r^{2}}{1-k r^{2}}+r^{2} d \theta^{2}+r^{2} \operatorname{sen}^{2} \theta d \varphi^{2},
$$

donde $a(t)$ es un factor de escala y $k$ es una constante que depende de la curvatura del espacio-tiempo y ha sido normalizada a los valores $-1,0,+1$ y describe un universo abierto, plano o cerrado respectivamente.

Una métrica modificada de Friedmann se obtiene introduciendo la función de lapse $\mathrm{N}(\mathrm{t})$, con esta consideración, la métrica de Friedmann toma la forma siguiente:

$d s^{2}=-N(t) d t^{2}+a^{2}(t) \frac{d r^{2}}{1-k r^{2}}+r^{2} d \theta^{2}+r^{2} \operatorname{sen}^{2} \theta d \varphi^{2}$.

Las variables $\mathrm{r}, \theta$ y $\varphi$ se denominan coordenadas comoviles, respecto a las cuales cualquier objeto estelar, como una galaxia, tiene valores fijos.

Por tanto el tensor métrico toma la forma:

$$
g_{\mu \nu}=\left(\begin{array}{cccc}
-N^{2}(t) & 0 & 0 & 0 \\
0 & \frac{a^{2}}{1-k r^{2}} & 0 & 0 \\
0 & 0 & a^{2} r^{2} & 0 \\
0 & 0 & 0 & a^{2} r^{2} \operatorname{sen}^{2} \theta
\end{array}\right)
$$

siendo los elementos covariantes y contravariantes del tensor métrico los siguiente

$g_{00}=-N^{2} \quad g_{11}=\frac{a^{2}}{1-k r^{2}} \quad g_{22}=a^{2} r^{2} \quad g_{33}=a^{2} r^{2} \operatorname{sen}^{2} \theta$

$g^{00}=-N^{-2} \quad g^{11}=\frac{1-k r^{2}}{a^{2}} \quad g^{22}=\frac{1}{a^{2} r^{2}} \quad g^{33}=\frac{1}{a^{2} r^{2} \operatorname{sen}^{2} \theta}$

(10)

\section{Componentes del tensor de Ricci y el escalar de Ricci}

Para el calculo de las componentes del tensor de Ricci debemos calcular primeramente las conexiones afín mediante los símbolos de Christoffel $\Gamma_{\mu \nu}^{\rho}$ los cuales están dados por:

$$
\Gamma_{\mu \nu}^{\rho}=\frac{1}{2} g^{\rho \sigma}\left[\frac{\partial g_{\sigma \mu}}{\partial x^{\nu}}+\frac{\partial g_{\sigma \nu}}{\partial x^{\mu}}-\frac{\partial g_{\mu \nu}}{\partial x^{\sigma}}\right]
$$

calculando el término $\Gamma_{o o}^{o}$ el cual viene dado por

$$
\Gamma_{o o}^{o}=\frac{1}{2} g^{o \sigma}\left[\frac{\partial g_{\sigma o}}{\partial x^{o}}+\frac{\partial g_{\sigma o}}{\partial x^{o}}-\frac{\partial g_{o o}}{\partial x^{\sigma}}\right]
$$

notamos que esta expresión tiene sentido cuando $\sigma=0$, para valores de $\sigma \neq 0$ la expresión $\Gamma_{o o}^{o}=0$, obteniéndose $\Gamma_{o o}^{o}=\frac{\dot{N}}{N}$. De forma similar calculamos las otras conexiones afín obteniéndose lo siguiente: 


$$
\begin{gathered}
\Gamma_{o o}^{o}=\frac{\dot{N}}{N} \\
\Gamma_{11}^{o}=\frac{a \dot{a}}{N^{2}\left(1-k r^{2}\right)} \\
\Gamma_{22}^{o}=\frac{r^{2} a \dot{a}}{N^{2}} \\
\Gamma_{33}^{o}=\frac{1}{N^{2}}\left(a \dot{a} \operatorname{sen}^{2} \theta\right) \\
\Gamma_{11}^{1}=\frac{k r}{\left(1-k r^{2}\right)} \\
\Gamma_{22}^{2}=\frac{\dot{a}}{a} \quad \Gamma_{12}^{1}=\frac{1}{r} \quad \Gamma_{33}^{2}=-\operatorname{sen} \theta \cos \theta \\
\left.\Gamma_{33}^{1}=-r\left(1-k r^{2}\right) \operatorname{sen}{ }^{2} \theta\right) \\
\frac{\dot{a}}{a} \quad \Gamma_{13}^{3}=\frac{1}{r} \quad \Gamma_{23}^{3}=-\cot \theta \cos \theta .
\end{gathered}
$$

El tensor de curvatura de Riemann permite determinar si una variedad tiene curvatura o no. De hecho, las variedades donde este tensor es nulo son las variedades planas. Se puede efectuar contracciones en el tensor de Riemann. El tensor de Ricci resulta de la contracción del tensor de Riemann y se puede construir a partir de la conexión afín (conexión de Levi Civita) y sus derivadas. Esta conexión es la que define la derivada covariante en una variedad métrica y por tanto nos dice como cambia un vector al moverlo paralelamente llevándolo de un punto a otro. Para el cálculo de las componentes del tensor de Ricci partimos de su definición, cuya expresión viene dada por:

$$
R_{\mu \nu}=\partial_{\alpha} \Gamma_{\mu \nu}^{\alpha}-\partial_{\nu} \Gamma_{\mu \alpha}^{\alpha}+\Gamma_{\mu \nu}^{\lambda} \Gamma_{\lambda \alpha}^{\alpha}-\Gamma_{\mu \alpha}^{\lambda} \Gamma_{\nu \lambda}^{\alpha}
$$

Calculando la componente $R_{00}$ la cual viene expresada por

$$
R_{00}=\partial_{\alpha} \Gamma_{00}^{\alpha}-\partial_{0} \Gamma_{0 \alpha}^{\alpha}+\Gamma_{00}^{\lambda} \Gamma_{\lambda \alpha}^{\alpha}-\Gamma_{0 \alpha}^{\lambda} \Gamma_{0 \lambda}^{\alpha}
$$

haciendo variar los indices $\alpha$ y $\alpha$ de 0 a 3, obtenemos $R_{00}=-3\left(\frac{\ddot{a}}{a}-\frac{\dot{N} \dot{a}}{N a}\right)$. Similarmente calculamos las demás componentes, obteniéndose lo siguiente:

$$
\begin{gathered}
R_{00}=-3\left[\frac{\ddot{a}}{a}-\frac{\dot{N} \dot{a}}{N a}\right] \\
R_{11}=\left[\frac{2 \dot{a}^{2}}{N^{2}}+\frac{a \ddot{a}}{N^{2}}-\frac{a \dot{a} \dot{N}}{N^{3}}+2 k\right]\left(\frac{1}{1-k r^{2}}\right)
\end{gathered}
$$

$$
\begin{array}{r}
R_{22}=\left(\frac{a \ddot{a}}{N^{2}}+\frac{2 \dot{a}^{2}}{N^{2}}-\frac{a \dot{a} \dot{N}}{N^{3}}+2 k\right) r^{2} \\
R_{33}=\left(\frac{a \ddot{a}+2 \dot{a}^{2}}{N^{2}}+2 k-\frac{a \dot{a} \dot{N}}{N^{3}}\right) r^{2} \operatorname{sen}^{2} \theta
\end{array}
$$

La escalar de Ricci resulta de la contracción del tensor de Ricci y viene definido:

$$
R=g^{\mu \nu} R_{\mu \nu}
$$

por lo tanto

$$
R=g^{00} R_{00}+g^{11} R_{11}+g^{22} R_{22}+g^{33} R_{33}
$$

reemplazando las expresiones (10),(19),(20),(21) y en (24) obtenemos el escalar de Ricci

$$
R=\frac{6}{a^{2} N^{3}}\left[N\left(\ddot{a} a+\dot{a}^{2}\right)-\dot{a} \dot{N} a+k N^{3}\right]
$$

\section{Formulación lagrangiana de las ecuaciones de Friedmann}

Usando la densidad lagrangiana de Einstein-Hilbert

$$
\sqrt{g R}=\sqrt{g} \frac{6}{a^{2} N^{3}}\left[N\left(\ddot{a} a+\dot{a}^{2}\right)-\dot{a} \dot{N} a+k N^{3}\right]
$$

definiendo como elemento de volumen como: $\sqrt{\tilde{g}}=\frac{\sqrt{g}}{a^{3} N}$, entonces obtenemos.

$\sqrt{g R}=a^{3} N \sqrt{\tilde{g}} R=\sqrt{\tilde{g}} \frac{6 a}{N^{2}}\left[N\left(\ddot{a} a+\dot{a}^{2}\right)-\dot{a} \dot{N} a+k N^{3}\right]$

considerando el lagrangiano gravitacional unidimensional

$$
L_{E H}=\frac{6 a}{N^{2}}\left[N\left(\ddot{a} a+\dot{a}^{2}\right)-\dot{a} \dot{N} a+k N^{3}\right]
$$

depende de $a(t), \dot{a}, \ddot{a}, N(t) y \dot{N}$. Para eliminar el término $\ddot{a}$ integramos por partes el primer término de $L_{E H}$

$$
\int 6 a^{2} N^{-1} \frac{d \dot{a}}{d t}=6 a^{2} N^{-1} \frac{\dot{a}}{d t}-\int \dot{a} \frac{d\left(6 a^{2} N^{-1}\right)}{d t}
$$

tomando el diferencial a la expresión anterior, esta queda como

$6 a^{2} \ddot{a} N^{-1}=\frac{d}{d t}\left(6 a^{2} \dot{a} N^{-1}\right)+6 a^{2} N^{-2}\left(-2 \dot{a}^{2} N+a \dot{a} \dot{N}\right)$.

Reemplazando el $L_{E H}$ obtenemos

$$
L_{E H}=\frac{6 a}{N^{2}}\left(-N \dot{a}+k N^{3}\right)+\frac{d}{d t}\left(6 a^{2} \dot{a} N^{-1}\right) .
$$


Seguidamente procedemos a calcular el tensor de curvatura extrínseca, el cual esta definido por

$$
K_{i j}=\frac{1}{2 N} \partial_{t} h_{i j}
$$

reemplazando los $h_{i j}$ obtenemos

$$
K_{i j}=\frac{\dot{a}}{N a} h_{i j}
$$

La traza del tensor de curvatura extrínseca viene definida por

$$
K=h^{i j} K_{i j}
$$

Reemplazando los valores de $h^{i j}$ y $K_{i j}$ obtenemos

$$
K=\frac{3 \dot{a}}{N a} .
$$

Introduciendo el término de frontera de GibbonsHawking-York [8]; y considerando el elemento de volúmen $\sqrt{\tilde{g}}$ y $\varepsilon=-1$ por tratarse de una hipersuperficie tipo espacial

$$
2 \varepsilon \sqrt{h} K \rightarrow L_{G H Y}=-2 a^{3}\left(\frac{3 \dot{a}}{a N}\right)=-6 a^{2} \dot{a} N^{-1} .
$$

La acción gravitacional será

$$
S_{g}\left[g_{\alpha \beta}\right]=S_{E H}\left[g_{\alpha \beta}\right]+S_{G H Y}\left[g_{\alpha \beta}\right] .
$$

El lagrangiano será

$$
\begin{gathered}
L_{g}=L E H+\frac{d}{d t} L_{G H Y} \\
L_{g}=6 N\left(-a\left(\frac{\dot{a}}{N}\right)^{2}\right)+K a
\end{gathered}
$$

Introduciendo la constante cosmológica: $\sqrt{g} R \rightarrow$ $\sqrt{g}(R-2 \Lambda)$ por lo tanto

$$
L_{g}=L_{A D M}=6 N\left(-a\left(\frac{\dot{a}}{N}\right)^{2}+K a-\frac{\Lambda a^{3}}{3}\right)
$$

Considerando un lagrangiano de materia asociado a un campo escalar $\phi$. Considerando la siguiente acción para un campo escalar con un potencial $V(\phi)$

$$
S\left[\phi, g_{\alpha \beta}\right]=\int \sqrt{g} d^{4} x\left[-\frac{1}{2} g^{\alpha \beta} \partial_{\alpha} \phi \partial_{\beta} \phi-V(\phi)\right] .
$$

Considerando la homogeneidad espacial requiere que el campo escalar, no dependa de coordenadas espaciales, solamente dependa del tiempo, entonces

$$
S\left[\phi, g_{\alpha \beta}\right]=\int a^{3} N \sqrt{\tilde{g}}\left[-\frac{1}{2} g^{00} \partial_{0} \phi \partial_{0} \phi-V \phi\right] d^{4} x
$$

$$
S\left[\phi, g_{\alpha \beta}\right]=\int \sqrt{\tilde{g}} d^{4} x N a^{3}\left(\frac{\dot{\phi}^{2}}{2 N^{2}}-V(\phi)\right) .
$$

Por lo tanto el lagrangiano total es

$$
L_{T O T}=\frac{1}{16 \pi G_{N}} L_{g}+L_{m}
$$

$$
\begin{array}{r}
L_{T O T}=N\left(-\frac{3}{4 \pi G_{N}} \frac{a \dot{a}^{2}}{2 N^{2}}+\frac{a^{3} \dot{\phi}^{2}}{2 N^{2}}\right. \\
\left.+\frac{3 K a}{8 \pi G_{N}}-\frac{\Lambda a^{3}}{8 \pi G_{N}}-a^{3} V(\phi)\right) .
\end{array}
$$

La acción $S_{T O T}[a, \phi, N]=\int d t L_{T O T}$ queda como

$$
\begin{array}{r}
S_{T O T}[a, \phi, N]=\int d t\left(-\frac{3}{4 \pi G_{N}} \frac{a \dot{a}^{2}}{2 N^{2}}+\frac{a^{3} \dot{\phi}^{2}}{2 N^{2}}+\frac{3 K a}{8 \pi G_{N}}\right. \\
\left.-\frac{\Lambda a^{3}}{8 \pi G_{N}}-a^{3} V(\phi)\right) .
\end{array}
$$

\section{Formulación hamiltoniana de las ecuaciones de Friedmann}

Partiendo del $L_{T O T}$ y considerando a $\Lambda$ dentro de $V(\phi)$, entonces $L_{T O T}$ queda como

$L_{T O T}=N\left(-\frac{3}{4 \pi G_{N}} \frac{a \dot{a}^{2}}{2 N^{2}}+\frac{a^{3} \dot{\phi}^{2}}{2 N^{2}}+\frac{3 K a}{8 \pi G_{N}}-a^{3} V(\phi)\right)$.

Considerando la variable $Q^{A}=\left(a_{(t)}, \phi_{(t)}\right)=(a, \phi)$ y un sistema bidimensional con métrica

$$
G_{A B}=\operatorname{diag}\left(\frac{-3 a}{4 \pi G_{N}}, a^{3}\right)
$$

obtenemos

$$
L_{T O T}=N\left(\frac{G_{A B}}{a N^{2}} \dot{Q_{A}} \dot{Q_{B}}-V(Q)\right),
$$

donde $V_{Q}=a^{3} V(\phi)-\frac{3 K a}{8 \pi G_{N}}$. Los momentos conjugados de las variables $Q^{A}$ son

$$
P_{A}=\frac{\partial}{\partial \dot{Q}^{A}} N\left(\frac{1}{2 N^{2}} G_{A B} \dot{Q}^{A} \dot{Q}^{B}-V(Q)\right) .
$$

Esto es

$$
\begin{gathered}
P_{a}=\frac{1}{N} G_{A A} \dot{Q}^{A}=-\frac{3 a \dot{a}}{4 \pi G_{N} N}, \\
P_{\phi}=\frac{1}{N} G_{B B} \dot{Q}^{B}=-\frac{a^{3} \dot{\phi}}{N},
\end{gathered}
$$

también 


$$
P_{N}=\frac{\partial L_{T O T}}{\partial \dot{N}}=0
$$

Construyendo el hamiltoniano, por definición tenemos

$$
H_{T O T}=P_{N} \dot{N}+P_{A} \dot{Q}^{A}-L_{T O T},
$$

obteniéndose

$$
H_{T O T}=N H(P, Q)=N\left(\frac{1}{2} G^{A B} P_{A} P_{B}+V(Q)\right),
$$

o en su forma equivalente

$$
H_{T O T}=N H=\frac{G_{A B}}{2 N} \dot{Q^{A}} \dot{Q}^{B}+N V(Q) .
$$

de donde se obtiene

$$
H=\frac{G_{A B}}{2 N^{2}} \dot{Q}^{A} \dot{Q}^{B}+V(Q) .
$$

La ecuación (57) representa la formulación hamiltoniana de la métrica de Friedmann. El formalismo desarrollado se puede aplicar a diferentes modelos cosmológicos; y el resultado obtenido proporciona un nuevo enfoque para estudiar la cosmología cuántica, que es un tema intrigante en la investigación teórica de la física. La ecuación (58) nos describe un sistema cosmológico en términos de variables posición y momento; el cual puede relacionarse, en cosmología cuántica, con el operador asociado con la energía del universo.

\section{Conclusiones}

Mediante el uso de la formulación ADM se ha logrado calcular la formulación lagrangiana de la métrica de Friedmann encontrandose que el tensor de curvatura extrínseca y su traza son directamente proporcionales a la velocidad del parámetro de escala, tambien se ha logrado calcular el lagrangiano total como resultado de un lagrangiano gravitacional y un lagrangiano de la materia. El formalismo ADM es una herramienta de mucha importancia en Relatividad General pues ha sido capaz de revelarnos la estructura hamiltoniana de la métrica de Friedmann.

\section{Referencias}

[1] A. Einstein. Preuss. Akad. Wiss. Berlin, Sitzber, 844 (1915).

[2] P. Dirac, A. Einstein. Preuss. Akad. Wiss. Berlin, Sitzber, 142 (1917).

[3] W. de Siter. Proc. Akad. Weteusch. amsterdam 19,1217 (1917).

[4] A. Friedmann. Phys.10,377 (1922);21, 326 (1924).

[5] P. Dirac. Proc. Roy. Soc.A246, P. 333-343, (1958).
[6] R. Arnowitt, S. Deser, C. Misner. Gravitation: an introduction to current research, editado por L. Witten (Ed. Wiley and Sons, Nova York, 1962).

[7] M. Ryan. Hamiltonian Cosmology, New York: Springer, 1972.

[8] G. Gibbons and S. Hawking, Action Integrals and Partition Functions in Quantum Gravity, Phys.Rev. D15 2752-2756, (1977).

[9] J. Tejeiro. Principios de relatividad general, Universidad Nacional de Colombia, 2005.

[10] S. Weinberg. Cosmology, Oxford University Press, 2008. 
